\title{
ADSORÇÃO DE ÍONS DE CROMO (VI) EM NANOFIBRAS DE QUITOSANA E NYLON 6 PRODUZIDAS POR TECNOLOGIA FORCESPINNING®
}

\author{
C. SCHONHART ${ }^{1}$, G. KUMMER ${ }^{1}$, G. L. DOTTO ${ }^{1}$, D. BERTUOL ${ }^{1}$ e E. H. TANABE ${ }^{1}$. \\ ${ }^{1}$ Laboratório de Processos Ambientais (LAPAM), Departamento de Engenharia Química, \\ Universidade Federal de Santa Maria, UFSM. \\ E-mail para contato: camila.schonhart@hotmail.com
}

\begin{abstract}
RESUMO - Este estudo teve como objetivo verificar o desempenho do biopolímero quitosana em associação com o nylon 6 como adsorvente para remoção de $\mathrm{Cr}$ VI presente em soluções aquosas. A partir dos experimentos de adsorção em batelada, foram avaliados os efeitos do $\mathrm{pH}$ e o ajuste dos dados experimentais aos modelos teóricos de Langmuir e Freundlich para determinar a capacidade máxima de adsorção do adsorvente estudado. A adsorção foi favorecida a $55^{\circ} \mathrm{C}$ e pH 3 com capacidade máxima de adsorção de $23,9 \mathrm{mg} \mathrm{g}^{-1}$.
\end{abstract}

\section{INTRODUÇÃO}

Uma das grandes preocupações atuais diz respeito ao impacto ambiental negativo gerado pelas indústrias. Segundo Zongo et al. (2009), Cr VI tem sido usado por décadas em uma ampla gama de setores industriais como, por exemplo, no tingimento de couros, indústria madeireira, produção de fertilizantes, entre outros. Ainda, Zongo et al. afirma que o cromo hexavalente é conhecido por ser altamente tóxico e cancerígeno.

Um dos métodos utilizados para remoção de $\mathrm{Cr}$ (VI) de soluções aquosas é o de adsorção. Segundo Fida et al. (2015), a adsorção apresenta alta rentabilidade e eficiência, facilidade de recuperação e de reutilização. Atuando como possível adsorvente tem-se as nanofibras em associação com biopolímeros, tendo a quitosana, segundo Razzaz et al. (2016), demostrado grande potencial adsorvente. Um método de grande destaque atualmente utilizado para a produção de nanofibras é, segundo Padron et al. (2013), o Forcespinning®, que faz uso da força centrifuga, conferindo alto rendimento e facilidade de produção.

Perante este contexto, o objetivo do presente trabalho foi desenvolver adsorventes de nanofibras com quitosana/nylon 6 via tecnologia Forcespinning®, para remoção de cromo VI de soluções aquosas. Desse modo, espera-se produzir um material apto a atuar como meio de descontaminação de efluentes industriais que contenham cromo hexavalente.

\section{MATERIAIS E MÉTODOS}

\subsection{Matérias primas, soluções e reagentes}


A quitosana em pó (grau de desacetilação $85 \pm 3 \%$, peso molecular $150 \pm 7 \mathrm{kDa}$ e tamanho de partícula $72 \pm 8 \mathrm{~mm}$ ) foi obtida a partir de resíduos de camarão (Penaeus brasiliensis) seguindo as indicações de Moura et al. (2015). Nylon 6 (5 mm de peletes) foi adquirido a partir de Sigma-Aldrich (EUA). Os reagentes utilizados foram ácido fórmico (98\%) (Neon, Brasil), $\mathrm{NaOH}(99 \%)$ (cromoline, Brasil) e $\mathrm{HCl}$ (37\%) (Synth, Brasil). A solução aquosa contendo íons de Cr VI foi obtida através da dissolução do dicromato de potássio $\left(\mathrm{K}_{2} \mathrm{Cr}_{2} \mathrm{O}_{7}\right)(99 \%$ Merck, Alemanha) em água destilada.

\subsection{Preparação das nanofibras de quitosana/nylon 6 via tecnologia Forcespinning®}

A solução de quitosana/nylon 6 foi feita a partir da dissolução do ácido fórmico e polímero, utilizando um agitador magnético a temperatura de $313 \mathrm{~K}$ por aproximadamente 6 horas de agitação, seguindo indicações de Ghani et al. (2014). A quantidade de solução polimérica por batelada foi de $10 \mathrm{~g}$, sendo a proporção mássica de quitosana/nylon 6 de 0,5 $\mathrm{g}_{\text {quitosana }}+2 \mathrm{~g}_{\text {nylon } 6}$ enquanto a fração de ácido fórmico manteve-se em 7,5 g por batelada.

As nanofibras foram produzidas com o equipamento de Forcespinning® (Fiberio, L1000, EUA), demonstrado na Figura 1. A fieira do equipamento foi preenchida pela solução polimérica e conectada com agulhas de $30 \mathrm{G} \frac{1}{2} 2$ " em cada extremidade da fieira. As condições de operação do equipamento foram de uma rotação de $10000 \mathrm{rpm}$, temperatura de $298 \mathrm{~K}$ e tempo de 10 minutos. As condições experimentais foram determinadas por testes preliminares e de acordo com informações fornecidas pela fabricante.

Figura 1 - Equipamento para produção de nanofibras via Forcespinning®.

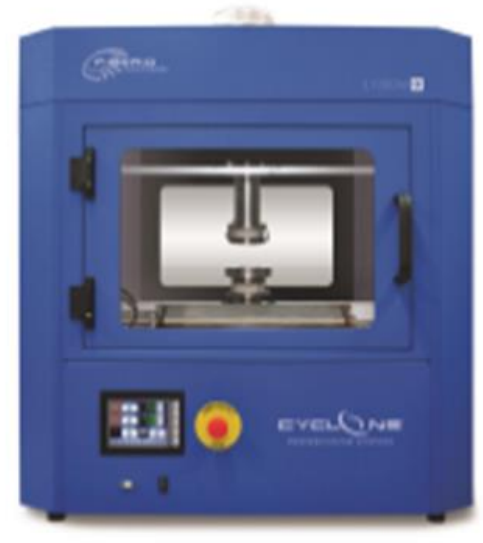

\subsection{Experimentos de adsorção}

Todos os experimentos de adsorção foram realizados com $50 \mathrm{~mL}$ de solução de cromo VI e $50 \mathrm{mg}$ de massa de nanofibras de quitosana/nylon $6 \mathrm{em}$ um agitador com termostato com rotação de 175 rpm (Marconi, MA 093, Brasil).

Inicialmente, investigou-se o efeito do $\mathrm{pH}$ (2-9) (ajustando as soluções com $\mathrm{NaOH}$ 0,1 M e $\mathrm{HCl} 1 \mathrm{M})$, para uma solução de cromo VI de $30 \mathrm{mg} \mathrm{L}^{-1}$ e agitação de 4 horas a $298 \mathrm{~K}$. 
Para as curvas de equilíbrio, foram utilizadas diferentes temperaturas $(298,308,318$ e $328 \mathrm{~K})$ e concentrações iniciais $\left(30,50,70,100,150\right.$ e $\left.200 \mathrm{mg} \mathrm{L}^{-1}\right)$, com melhor $\mathrm{pH}$ e agitação de 4 horas a $298 \mathrm{~K}$.

Após os experimentos as amostras foram filtradas e a solução aquosa foi quantificada pelo equipamento de Espectrometria de Adsorção Atômica com Chama (Agilent Technologies, modelo 200 Séries AA).

\section{EQUAÇÕES}

A porcentagem de remoção de cromo VI $(\mathrm{R} \%)$ e a capacidade de adsorção $\left(\mathrm{q}_{\mathrm{e}}\right)$ foram determinadas pelas Equações 1 e 2, respectivamente:

$$
\begin{aligned}
& R \%=\frac{\left(C 0-C_{\theta}\right)}{C_{0}} 100 \\
& q e=\frac{\left(C 0-C_{\theta}\right)_{\cdot} V}{m}
\end{aligned}
$$

Para representar as curvas de equilíbrio da adsorção de cromo VI pelas nanofibras de quitosana/nylon6 foram usados os modelos de Freundlich e Langmuir, representados pelas Equações 3 e 4, respectivamente:

$$
\begin{aligned}
& q_{e}=K_{F} C_{e}{ }^{1 / n} F \\
& q_{e}=q_{m} K_{L} C_{e} / 1+\left(K_{L} C_{e}\right)
\end{aligned}
$$

\section{RESULTADOS E DISCUSSÕES}

\subsection{Efeito do pH na adsorção de íons cromo VI}

A Figura 2 apresenta as capacidades de adsorção e os percentuais de remoção de cromo VI utilizando nanofibras de quitosana/nylon 6. Observa-se que a capacidade de adsorção e remoção é favorecida com a diminuição do $\mathrm{pH}$ da solução. Quando o $\mathrm{pH}$ diminuiu de 9 para 3 , a capacidade de adsorção e remoção das nanofibras aumentou de $4,3 \mathrm{mg} \mathrm{g}^{-1}$ e $16,8 \%$ para $6,4 \mathrm{mg} \mathrm{g}^{-1}$ e 26,4\%, tornando-se o pH 3 o ideal, como apresenta a Figura 2.

Figura 2 - Efeito do pH na capacidade de adsorção e remoção de cromo VI.

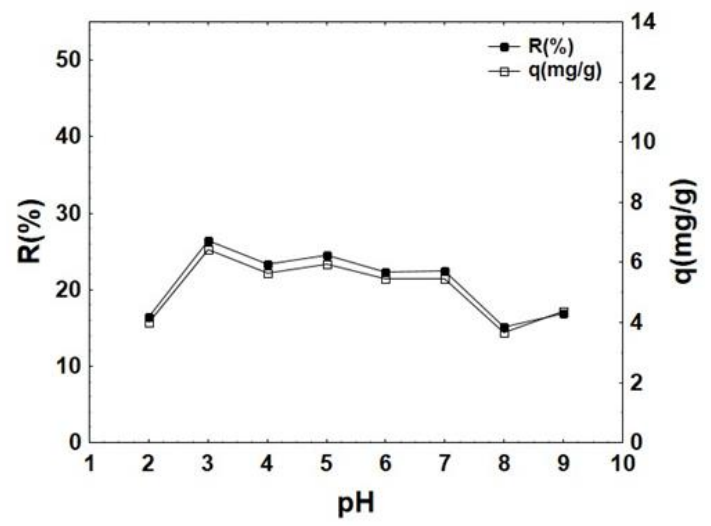


De acordo com Markiewicz et al. (2015), o cromo VI em soluções aquosas existe nas formas de ânions $\mathrm{HCrO}_{4}{ }^{-}, \mathrm{CrO}_{4}{ }^{2-}$ e $\mathrm{Cr}_{2} \mathrm{O}_{7}{ }^{2-}$ e podem ser adsorvidos por adsorventes carregados positivamente através de atração eletrostática. Os baixos valores de $\mathrm{pH}$ influenciam diretamente as cargas da superfície das nanofibras de quitosana/nylon 6 que tem em sua estrutura os grupos funcionais $-\mathrm{OH}$ e $\mathrm{NH}_{2}$. Em meio ácido seus grupos funcionais carregados positivamente protonados ligam-se aos ânions $\mathrm{HCrO}_{4}{ }^{-}, \mathrm{CrO}_{4}{ }^{2-}$ e $\mathrm{Cr}_{2} \mathrm{O}_{7}{ }^{2-}$. Conforme ocorre o aumento do $\mathrm{pH}$, a protonação desses grupos enfraquece diminuindo a capacidade de adsorção das nanofibras. Desse modo, o restante dos testes foi conduzido com pH 3 para as nanofibras de quitosana/nylon 6 .

\subsection{Isotermas de adsorção}

Os modelos das isotermas de Freundlich e Langmuir foram utilizados para descrever as curvas isotérmicas. A Tabela 1 mostra os valores dos parâmetros isotérmicos para adsorção de cromo VI pelas nanofibras de quitosana/nylon 6. A partir da avaliação dos altos valores do coeficiente de determinação $\left(\mathrm{R}^{2}\right)$ e valores baixos para o erro médio relativo (ARE), os dois modelos foram eficientes em explicar os dados coletados.

Tabela 2 - Parâmetros isotérmicos para adsorção de cromo VI

\begin{tabular}{ccccc}
\hline \multirow{2}{*}{ Modelo isotérmico } & \multicolumn{5}{c}{ Temperatura (K) } \\
\cline { 2 - 5 } & \multicolumn{4}{c}{ Nanofibras de quitosana/nylon 6} \\
\hline Modelo de Freundlich & & & \\
\hline $\mathrm{K}_{\mathrm{F}}\left(\mathrm{mg} \mathrm{g}^{-1}\right)\left(\mathrm{mg} \mathrm{L}^{-1}\right)^{-1 / \mathrm{n}} \mathrm{F}$ & 1.61 & 2.09 & 4.75 & 3.79 \\
\hline $1 / \mathrm{n}_{\mathrm{F}}$ & 0.414 & 0.386 & 0.239 & 0.332 \\
\hline $\mathrm{R}^{2}$ & 0.9961 & 0.9925 & 0.979 & 0.9952 \\
\hline $\mathrm{ARE}(\%)$ & 2.23 & 0.14 & 0.13 & 2.78 \\
\hline Modelo de Langmuir & & & & \\
\hline $\mathrm{q}_{\mathrm{m}}\left(\mathrm{mg} \mathrm{g}^{-1}\right)$ & 16.9 & 18.1 & 17.6 & 23.9 \\
\hline $\mathrm{K}_{\mathrm{L}}\left(\mathrm{L} \mathrm{mg}^{-1}\right)$ & 0.0194 & 0.0236 & 0.0492 & 0.0303 \\
\hline $\mathrm{R}_{\mathrm{L}}\left(\mathrm{C}_{0}=200 \mathrm{mg} \mathrm{L}^{-1}\right)$ & 0.205 & 0.1749 & 0.0923 & 0.1416 \\
\hline $\mathrm{R}^{2}$ & 0.9805 & 0.996 & 0.9943 & 0.9939 \\
\hline $\mathrm{ARE}(\%)^{5}$ & 5.5 & 1.94 & 2.54 & 2.43 \\
\hline
\end{tabular}

Apesar de ambos terem se ajustado de maneira satisfatória aos dados do experimento, optou-se pelo modelo de Langmuir, uma vez que este apresentou um maior ajustamento aos dados experimentais se comparado ao de Freundlich.

A máxima capacidade de adsorção de cromo VI pelas nanofibras de quitosana/nylon 6 nas condições experimentais estudadas foi de $23,9 \mathrm{mg} \mathrm{g}^{-1}$. 
A Figura 3 mostra as curvas isotérmicas de adsorção do cromo VI com nanofibras de quitosana/nylon 6 representadas pelo modelo de Langmuir. No modelo de Langmuir o valor de $\mathrm{R}_{\mathrm{L}}$ (parâmetro de equilíbrio) variou entre 0 e 1 , indicando que o processo de adsorção de cromo VI foi do tipo favorável para as nanofibras. Também, o aumento da temperatura de 298 até $328 \mathrm{~K}$ favoreceu a capacidade de adsorção de cromo VI pelas nanofibras, indicando que a adsorção de Cr VI nas nanofibras é endotérmica.

Figura 3 - Curvas isotérmicas de adsorção de cromo VI.

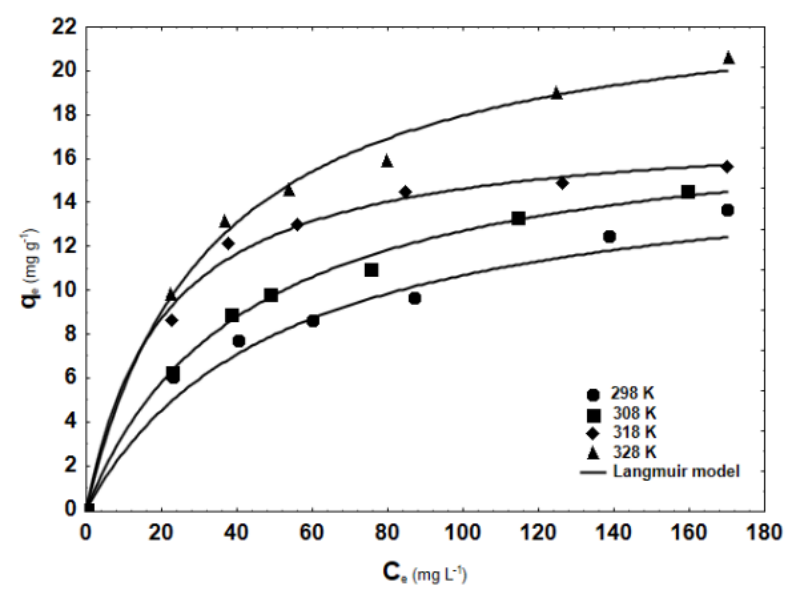

De acordo com os estudos de Li et al. (2012), há três fatores capazes de explicar o aumento da capacidade de adsorção com o aumento da temperatura. $\mathrm{O}$ primeiro fator seria a ampliação da dimensão dos poros e/ou a ativação da superfície adsorvente a temperaturas mais altas. $\mathrm{O}$ segundo fator seria o aumento no número de moléculas ativadas com o aumento da temperatura, ocasionando um aumento na frequência de colisões entre moléculas. $\mathrm{O}$ terceiro fator seria o incremento na mobilidade dos íons Cr VI presentes na solução em direção à superfície adsorvente e o consequente aumento da penetração dos íons dentro das nanofibras.

\section{CONCLUSÃO}

Tendo em vista o nocivo potencial de certos componentes presentes nos efluentes industriais, entre eles o metal pesado cromo hexavalente, desenvolveu-se neste trabalho um adsorvente composto por um biopolímero em associação com o nylon 6 capaz de atuar no tratamento deste resíduo. Os resultados mostraram que a adsorção de Cr VI é favorecida em pH 3 e a temperaturas elevadas. A adsorção de Cr VI utilizando as nanofibras de quitosana foi bem representada pelo modelo de Langmuir, sendo a máxima capacidade de adsorção de 23,9 miligramas de metal por grama de adsorvente, dada a $55{ }^{\circ} \mathrm{C}$. Tendo como base estes resultados, é possível afirmar que essas nanofibras são uma alternativa promissora no tratamento de efluentes.

\section{NOMENCLATURA}

$1 / \mathrm{n}_{\mathrm{F}}-$ fator de heterogeneidade;

$\mathrm{C}_{0}-$ concentração inicial $\left(\mathrm{mg} \mathrm{L}^{-1}\right)$; 
$\mathrm{C}_{\mathrm{e}}$ - concentração de equilíbrio $\left(\mathrm{mg} \mathrm{L}^{-1}\right)$;

$\mathrm{K}_{\mathrm{F}}-$ constante de Freundlich $\left(\mathrm{mg} \mathrm{g}^{-1}\right)\left(\mathrm{mg} \mathrm{L}^{-1}\right)_{\mathrm{F}}^{-1 / \mathrm{n}}$;

$\mathrm{K}_{\mathrm{L}}$ - constante de Langmuir;

$\mathrm{m}$ - massa de adsorvente $(\mathrm{g})$;

$\mathrm{q}_{\mathrm{m}}$ - capacidade máxima de adsorção $\left(\mathrm{mg} \mathrm{g}^{-1}\right)$;

V - volume da solução (L).

\section{REFERÊNCIAS BIBLIOGRÁFICAS}

FIDA, H.; GUO, S.; ZHANG, G.; Preparation and characterization of bifunctional Ti-Fe kaolinite composite for Cr (VI) removal. Journal of Colloid and Interface Science 442 (2015) 30-38.

GHANI, M.; GHAREHAGHAJI, A. A.; ARAMI, M.; TAKHTKUSE, N.; REZAEI, B.; Fabrication of Electrospun Polyamide-6/Chitosan Nanofibrous Membrane toward Anionic Dyes Removal. Journal of Nanotechnology. Volume 2014, Article ID 278418, 12 pages.

LI, W.; TANG, Y.; ZENG, Y.; TONG, Z.; LIANG, D.; CUI, W.; Adsorption behavior of Cr(VI) ions on tannin-immobilized activated clay. Chemical Engineering Journal 193194 (2012) 88-95.

MARKIEWICZ, B.; KOMOROWICZ, I.; SAJNÓG, A.; BELTER, M.; BARALKIEWICZ, D.; Chromium and its speciation in water samples by HPLC/ICP-MS - technique establishing metrological traceability: A review since 2000. Talanta 132 (2015) 814828.

MOURA, J. M.; FARIAS, B. S.; RODRIGUES, D. A. S.; MOURA, C. M.; DOTTO, G. L.; PINTO, L. A. A.; Preparation of Chitosan with Different Characteristics and Its Application for Biofilms Production. J. Polym. Environ. 23 (2015) 470-477.

PADRON, S.; FUENTES, A.; CARUNTU, D.; LOZANO, K.; Experimental study of nanofiber production through forcespinning. Journal of Applied Physics 113, 024318 (2013).

RAZZAZ, A.; GHORBANA, S.; HOSAYNI, L.; IRANI, M.; ALIABADI, M.; Chitosan nanofibers functionalized by $\mathrm{TiO}_{2}$ nanoparticles for the removal of heavy metal ions. Journal of the Twain Institute of Chemical Engineers 58 (2016) 333-343.

ZONGO, I.; LECLERC, J.; MAÏGA, H. A.; WÉTHÉ, J.; LAPICQUE, F.; Removal of hexavalent chromium from industrial wastewater by electrocoagulation: $A$ comprehensive comparison of aluminium and iron electrodes. 66 (2009) 159-166. 